\title{
Methods for pixel domain correction of EB leakage
}

Liu, Hao; Creswell, James; von Hausegger, Sebastian; Naselsky, Pavel

Published in:

Physical Review D

DOI:

10.1103/PhysRevD.100.023538

Publication date:

2019

Document version

Publisher's PDF, also known as Version of record

Citation for published version (APA):

Liu, H., Creswell, J., von Hausegger, S., \& Naselsky, P. (2019). Methods for pixel domain correction of EB leakage. Physical Review D, 100(2), [023538]. https://doi.org/10.1103/PhysRevD.100.023538 


\title{
Methods for pixel domain correction of $E B$ leakage
}

\author{
Hao Liu, ${ }^{1,2, *}$ James Creswell, ${ }^{1, \dagger}$ Sebastian von Hausegger, ${ }^{1,}$ and Pavel Naselsky ${ }^{1, \S}$ \\ ${ }^{1}$ The Niels Bohr Institute and Discovery Center, Blegdamsvej 17, DK-2100 Copenhagen, Denmark \\ ${ }^{2}$ Key laboratory of Particle and Astrophysics, Institute of High Energy Physics, \\ CAS, 19B YuQuan Road, 100049 Beijing, China
}

(Received 11 November 2018; published 25 July 2019)

\begin{abstract}
In the observation of the cosmic microwave background (CMB) polarization, " $E B$ leakage" refers to the artificial $B$-mode signal coming from the leakage of the $E$-mode signal when part of the sky is unavailable or excluded. Correction of such a leakage is one of the preconditions for detecting primordial gravitational waves via the $\mathrm{CMB} B$-mode signal. In this work, we design two independent methods for correcting the $E B$ leakage directly in the pixel domain using standard definitions of the $E$ - and $B$-modes. The two methods give consistent results, and both are fast and easy to implement. Tests on a CMB simulation containing a zero initial $B$-mode show an efficient suppression of the $E B$ leakage. When combined with the MASTER method to reconstruct the full-sky $B$-mode spectrum in simulations with a relatively simple mask, the error from EB-leakage is suppressed further by more than 1 order of magnitude at the recombination bump, and up to 3 orders of magnitude at higher multipoles, compared to a "pure" MASTER scheme under the same conditions. Meanwhile, although the final power spectrum estimation benefits from apodization, the pixel domain correction itself is done without apodization, and thus the methods offer more freedom in choosing an apodization based on specific requirements.
\end{abstract}

DOI: 10.1103/PhysRevD.100.023538

\section{INTRODUCTION}

The analysis of the cosmic microwave background (CMB) polarization lies in the focus of current $\mathrm{CMB}$ missions such as Planck, as well as future missions [1-8]. The main scientific goal is to detect primordial gravitational waves in the $B$-mode of the polarized CMB signal. The contribution of primordial gravitational waves is quantified by the tensor-to-scalar ratio $r$. Currently, this parameter is constrained to $r \lesssim 0.07[9,10]$, and future missions are aiming for a sensitivity of $r \sim 10^{-4}$ [5]. However, CMB missions of the near future are all ground-based, which means that they provide only partial sky coverage. On an incomplete sky map, the separation of the polarized signal into $E$ - and $B$-modes will be affected by "leakage" (the socalled $E B$ leakage), and the resulting $B$ map can be strongly contaminated $[11,12]$. This kind of leakage must be carefully corrected to reach the above target.

One way to study the $E B$ leakage due to incomplete sky coverage is by constructing localized estimators that are associated with the mask or window function defined on the sky fraction in question, as was first proposed by [12], and subsequently used in many such studies, e.g., [13-18]. Commonly such estimators are referred to as

\footnotetext{
"liuhao@nbi.dk

james.creswell@nbi.ku.dk

s.vonhausegger@nbi.dk

§naselsky@nbi.dk
}

"pure" $E / B$-modes. However, note that so far it was not noticed how to perform a pixel domain conversion from the "pure" $E$ - and $B$-modes to standard $E$ - and $B$-modes that are defined on the full sky. It is important to note that only such full-sky $E$ - and $B$-modes are always orthogonal to each other.

We here introduce two methods for the correction of the $E B$ leakage in the pixel domain, which only use the standard full-sky definitions of $E$ - and $B$-modes [19-21]. The first method is motivated by studying properties of the leakage at the mask's boundary: in a series of works [14,22-24], the technical details of the $E B$ leakage and their possible solutions were thoroughly discussed, and even the idea of correcting the leakage using relaxation methods was mentioned. In our paper, we implement the relaxation method using diffusive inpainting, e.g., [25,26]. However, we also note the limitation of the relaxation method: it only gives a particular solution that could ignore small scale features. Improvement of this solution requires more knowledge about the real $B$-mode signal inside the region, which is unfeasible in this way.

To this effect, we introduce an alternative and novel $E B$ leakage correction method in the pixel domain. The $E$ and $B$ signals are regarded as being composed of contributions from different regions of the sky. When part of the sky is unavailable the leakage correction is carried out by recycling the $E$-family component of the $Q$ and $U$ Stokes parameters [27] derived from only the available sky region. 
The two methods give similar results. We show that generally the second method performs better, and we will therefore focus our attention on it. However, in special cases the first method can outdo the latter as we discuss below.

We emphasize that neither of the two methods requires prior knowledge of the underlying $E E$ or $B B$ power spectra, which is of significant advantage. It is also important to note that, compared to previous methods, e.g., [16-18,28], this work provides the first correction of the $E B$ leakage in the pixel domain using the standard full-sky definition of the $E$ - and $B$-modes. We shall discuss a list of benefits in the main body of this paper.

This paper is organized as follows: in Sec. II, we introduce the two methods and provide examples using two different masks for illustration. Their performance is tested in Sec. III, and a brief discussion is given in Sec. IV.

\section{METHODS AND EXAMPLES}

We here introduce two independent methods; the first is a relaxation method, and the second provides a more elaborate solution utilizing our recently introduced $E B$ families. In [29], we provide mathematical proof that method 2 is the best blind correction of the EB-leakage in the pixel-domain, but in this work we still present the results of both methods as a useful cross-check.

For the convenience of reading, the background and principles of the two methods will be introduced in the Appendix B, and below we present the procedures of the two methods directly.

\section{A. Method 1: Diffusive inpainting}

Method 1 is to estimate the $E B$-leakage by diffusive inpainting $[25,26]$, in which sky pixels ${ }^{1}$ are iteratively replaced by the average of their neighbors, except for the pixels on the boundary. The procedure is

(1) Begin with the corrupted $B$ map derived from a masked sky (for calculation of the $B$ map, see Appendix A 1).

(2) Set all pixels on the sky to zero except those at the edge of the valid region, which constitute the boundary condition.

(3) Perform diffusive inpainting on the valid sky as mentioned above using the boundary condition in step 2. On convergence, the result is a template for the $E B$ leakage.

(4) Subtract the derived template from the corrupted $B$ map in order to arrive at the corrected $B$ map.

\footnotetext{
${ }^{1}$ For this we have used the HEALPIX package (http://healpix .sourceforge.net) and therefore adopted their pixelization scheme. However, the method is not tailored to function with that pixelization only.
}

\section{B. Method 2: Recycling the E-mode}

Method 2 is to estimate the $E B$-leakage by recycling the $E$-mode signal. As mentioned above, this is the best blind estimate of the EB-leakage in pixel domain. The procedure is

(1) Begin with a sky map $\mathbf{P}=(Q, U)$ and a mask, calculate $\mathbf{P}_{\mathbf{E}}^{\prime}=\left(Q_{E}, U_{E}\right)^{\prime}$ and $\mathbf{P}_{\mathbf{B}}^{\prime}=\left(Q_{B}, U_{B}\right)^{\prime}$ directly from masked $\mathbf{P}$.

(2) Similarly, obtain $\mathbf{P}_{\mathbf{B}}^{\prime \prime}=\left(Q_{B}, U_{B}\right)^{\prime \prime}$ from masked $\mathbf{P}_{\mathbf{E}}{ }^{\prime}$.

(3) Using the same mask, $\mathbf{P}_{\mathbf{B}}^{\prime \prime}$ is the template for the $E B$ leakage in the available region. Use it to remove the $E B$ leakage from $\mathbf{P}_{\mathbf{B}}{ }^{\prime}$ by linear fitting.

Above we only described the method and the procedure in terms of the $E$ - and $B$-families. Note that while it is necessary to do step 1 via the $E$ - and $B$-family decomposition, starting from step 2 , one is also free to proceed in terms of the actual $B$-modes, and arrive at a $B$ map as a template; both give similar results. However, for power spectrum estimation, the $B$ map template gives slightly better results (about $10 \%$ lower error). For correcting the morphology of the corrupted map in the pixel domain, the variant with $\left(Q_{B}, U_{B}\right)$ " is slightly better. In this work, since we will eventually compute power spectra, all pixel domain results will be presented in the form of $B$ maps.

\section{Examples and comparison}

We now present examples of correcting the $E B$ leakage on simulated CMB maps with two different masks, shown in Figs. 1 and 2. For this purpose we select a simulated CMB map with $r=0.05$ from Planck's FFP9 suite. Both figures show the true signals in row 1 for reference and the results of correction in rows 2 and 3.

The belt region shown in Fig. 1 was defined to be $20^{\circ}$ in width and $2^{\circ}$ in height. The two methods give similar leakage templates, and additionally reproduce the real leakage term well. As a measure of similarity, we compute the cross-correlation between the real $B$ map and the contaminated one to be only $20 \%$, whereas after correction, the $B$ map corrected by method 1 gives $86 \%$ correlation with the real $B$ map, and that of method 2, 66\%. While method 1 leads to better correction on larger scales, method 2 captures the small scale leakage better, as can be seen in the right panels of rows 2 and 3 .

We then repeat this test by instead using a disk-shaped region with $20^{\circ}$ radius as shown in Fig. 2 . This time, the cross-correlation between the real $B$ map and the contaminated one is $70 \%$, whereas after correction, method 1 leads to $97.7 \%$ correlation of the fixed $B$ map with the real one, and that of method 2 gives $97.6 \%$, in strong agreement with one another, as well as with the real $B$ map. A glance at the figures makes clear that most of the interior of the map is significantly contaminated, which is captured well by the templates. Given the small fraction of the edge area in comparison to the whole region, the cross-correlations are 
Real leakage term

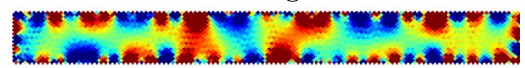

Template (method 1$)$

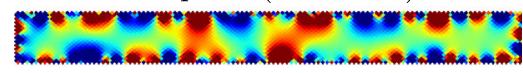

Template (method 2)

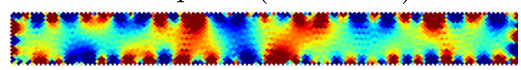

Real B map

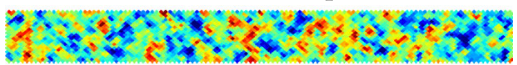

Fixed B map (method 1)

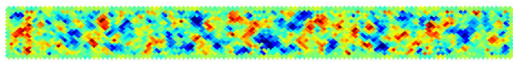

Fixed B map (method 2)

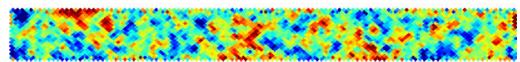

Corrupted B map

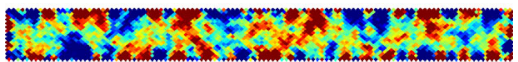

Residual (method 1)

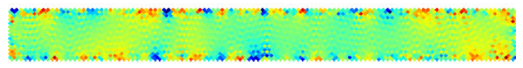

Residual (method 2)

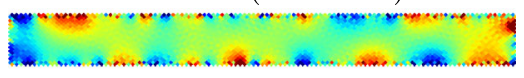

FIG. 1. Examples of pixel domain $E B$ leakage corrections with a $r=0.05$ simulation in a belt region that is $20^{\circ}$ wide and $2^{\circ}$ high. Upper panels: The real leakage term (left), the real $B$ map (middle), and the corrupted $B$ map (right). Middle panels: The results of method 1. The derived template (left), the corrected $B$ map (middle), and the residual leakage (right). Bottom panels: Same as middle panels but for method 2 .

only marginally influenced by the edge, especially after correction. Also note that the cross-correlations are associated with a given mask, and are not comparable across masks.

Further tests will show that method 2 gives relatively smaller error at the desired multipole range (as elaborated in Sec. III C), whereas method 1 mainly involves the correction of the large-scale features (see also the smoothness of the template by method 1 in Fig. 1). Therefore, method 2 will be the default method for the rest of this work. However, as was the purpose of this section, we point out that method 1 can perform better in the case of narrow regions, where the edge condition becomes relatively more important.
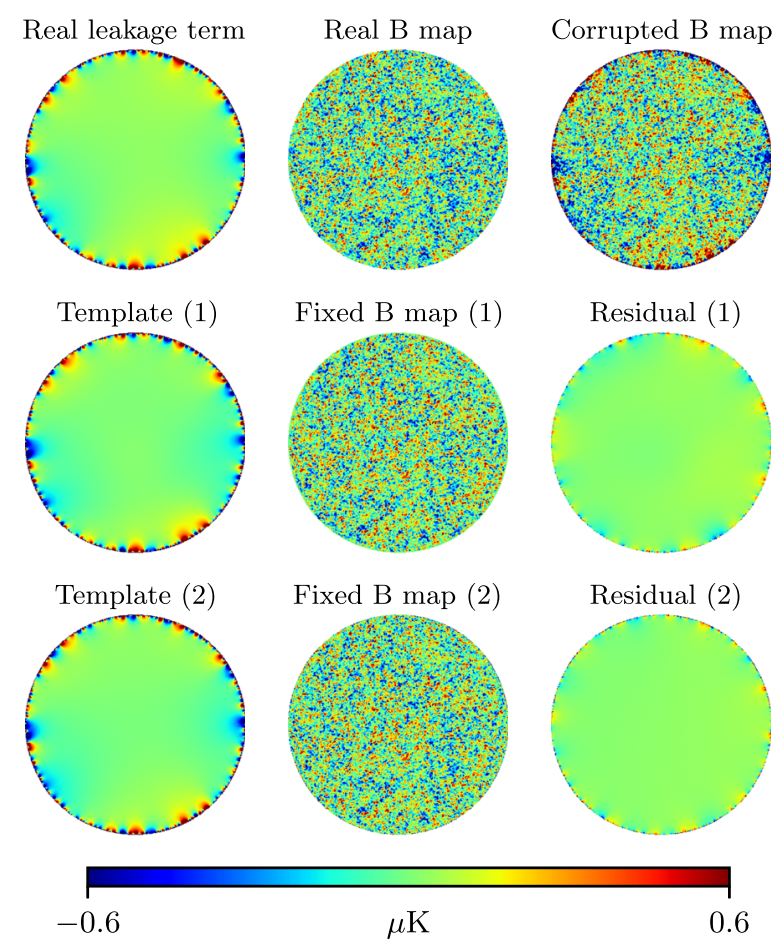

$\mu \mathrm{K}$

\section{Advantages of correction in pixel domain}

Concluding this section, we summarize the advantages a correction of $E B$ leakage in the pixel domain has over conventional methods that only recover the $E E$ and $B B$ power spectra.

(i) Both methods 1 and 2 operate only in the pixel domain without involving the power spectrum, i.e., they are independent of assumptions on the $B$-mode angular power spectrum, and therefore should be considered an additional contribution to existing polarized power spectrum reconstruction methods.

(ii) As will be shown, another important advantage of pixel domain correction is that it is very easy to deal with noise, because there noise and CMB are added linearly, and our proposed correction methods are also linear.

(iii) Since we have already corrected the $E B$ leakage in the pixel domain, the challenge to arrive at an estimation of the $E$ - or $B$-mode power spectrum simplifies to estimating the angular power spectrum of a scalar field given a mask. This problem has been intensively studied by many authors, e.g., [30-47]. This idea is implemented in Sec. III B, which gives an excellent reconstruction result.

(iv) As was seen, neither method 1 nor 2 requires any apodization of the mask; they work simply with a top-hat mask. One is thus free to choose any posterior apodization scheme to improve the $B$-mode angular power spectrum estimation. This will be presented in Sec. III C.

\section{TESTING THE LEVEL OF RESIDUAL AFTER CORRECTION}

Even with a perfect $E B$ leakage correction, the $B$-mode spectrum obtained from the cut sky is still different from the known full-sky spectrum, due to sampling uncertainty (among others). To focus on the effectiveness of our

FIG. 2. Same as Fig. 1 but for a disk mask with $20^{\circ}$ radius. 
methods, in this section, we perform tests that measure which uncertainties to expect in $B$-mode power spectra only from the contribution of the $E B$ leakage or its correction. We hereto use CMB simulations from the FFP9 suite [48], which include the scalar, tensor and non-Gaussian components, as well as a correctly simulated lensing effect. As before, we select those with a tensor-to-scalar ratio $r=0.05$ (except for Sec. III A). For all the tests we will investigate a disk-shaped sky region of about $47^{\circ}$ radius, covering roughly $15 \%$ of the sky. This choice was made with reference to one of the specifications of the GreenPol experiment $[49,50]$.

First, in Sec. III A, we perform a null test on a zero$B$-mode simulation. We then move on to investigate simulations with nonzero $B$-modes and compare our results to those obtained from "purifying" the $E$ - and $B$-modes in Sec. III B. Lastly, we illustrate how to further optimize these results by different choices of posterior apodization in Sec. III C.

\section{A. Zero initial B-mode}

We begin with an idealistic test, in which we select a single simulated CMB map without noise, and manually set the input $B$-mode to zero. This automatically marks any detection of a derived $B$-mode signal — either before or after correction - to be due to leakage or residual leakage. After masking, we attempt to perform an $E / B$ decomposition and subsequently use the recycling method for the correction of the corrupted $B$ map. We compare this to a case where no correction has been done to the corrupted maps. The final output $B$-mode spectra are then calculated directly from the masked maps, in two ways: once where the maps were apodized with a Tukey window for which we used a taper fraction of $0.1,{ }^{2}$ and once where they were not. Those that were apodized were rescaled such that the spectra are comparable. These spectra correspond to pseudospectra and, as we mentioned before, are sufficient for highlighting the advantages of our method, without including sample uncertainties. As pointed out by [12], oversampling can help to reduce the leakage due to pixelization; thus we use $N_{\text {side }}=2048$ in this test, and show the results in Fig. 3. In the upper panels it can be seen that the leakage from $E$ - to $B$-modes is removed almost completely. (We amplify the residuals by a factor of 10 to make them visible.) In the bottom panel we show the angular power spectra of the residual leakages before (red) and after (blue) correction. One can see that those whose maps were apodized (dashed lines) generally give better results than those which were not (solid lines). The corrected and apodized spectrum gives the best result, which lies up to 12 orders of magnitude below the input $E E$ spectrum. The other variants

\footnotetext{
${ }^{2}$ This roughly corresponds to an apodization length of $5^{\circ}$. In Sec. III C we study different window functions for apodization, including the one used here.
}
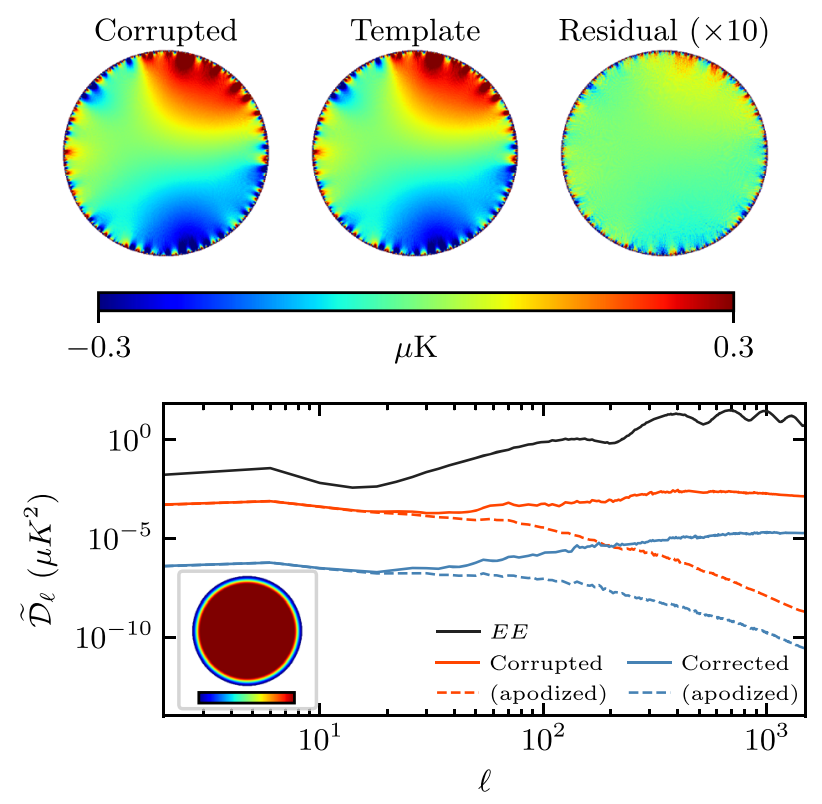

FIG. 3. Maps and power spectra after $E B$ leakage correction when the input $B$-mode is zero. Upper panels: The corrupted $B$-mode (left), the template generated by the recycling method (middle), and the residual leakage after correction (multiplied by 10; right). Lower panel: Comparison of the $E E$ pseudospectrum (black), and the residual $B B$ pseudospectra (both binned with $\Delta \ell=4$ ) derived from either corrupted (red) or corrected (blue) $B$ maps, using either the mask (solid) or apodization shown in the inset (dashed).

are either worse at large scales (the corrupted $B B$ spectra), or worse at small scales (without posterior apodization), or both. We already here refer to Sec. III C, where we show that our result can be further improved by about 2 orders of magnitude by optimizing the apodization.

\section{B. Combination with the MASTER method}

We now extend above test, in which we only considered $B B$ pseudospectra, to the reconstruction of full $B$-mode spectra. A widely used algorithm to reconstruct an unbiased full-sky angular power spectrum from the cut sky is the MASTER method [32]. Our pixel domain EB leakage correction can be easily combined with the MASTER method (or any other pseudo- $C_{\ell}$ method) in the following way.

We hereto use the Python package, PYMASTER, of the NAMASTER code $[51,52]$ as an implementation of the MASTER method to reconstruct the full-sky $B B$ spectrum by two ways for comparison: one is by using NAMASTER with a built-in purifying [17] option for the $B$-mode, ${ }^{3}$ whose results are denoted $\left(C_{\ell}^{B B}\right)_{1, i}$ for 50 different simulations $i$, and will in the following be referred to as "MASTER + PURE"; and the second is to first correct

\footnotetext{
${ }^{3}$ http://namaster.readthedocs.io/en/latest/sample_pureb.html.
} 
the $B$-mode map by our recycling method, and subsequently use NAMASTER in the nonpolarized mode to reconstruct the full-sky $B B$ spectrum from the corrected $B$ map as $\left(C_{\ell}^{B B}\right)_{2, i}$. Lastly, we run MASTER on the real $B$ map for each simulation masked with the same apodization to provide a reference $\left(C_{\ell}^{B B}\right)_{0, i}$. This helps to skip the sampling uncertainty and focus only on the error of $E B$ leakage correction. The MASTER reconstructions start from $\ell=16$ and the bin size is also 16; thus the first bin is centered at $\ell=24$. For each simulation we calculate the differences between the reconstructions and the reference; subsequently for each we compute the corresponding RMS and normalized average offsets as

$$
\begin{gathered}
\Delta_{1,2}^{i}(\ell)=\left(C_{\ell}^{B B}\right)_{(1,2), i}-\left(C_{\ell}^{B B}\right)_{0, i} ; \\
\Delta_{1,2}(\ell)=\sqrt{\frac{1}{N_{\text {sim }}} \sum_{i=1}^{N_{\text {sim }}}\left[\Delta_{1,2}^{i}(\ell)\right]^{2},} \\
\epsilon_{1,2}(\ell)=\frac{\left\langle\Delta_{1,2}^{i}(\ell)\right\rangle}{\left\langle\left(C_{\ell}^{B B}\right)_{0, i}\right\rangle} .
\end{gathered}
$$

We plot $\Delta_{1}(\ell)$ and $\Delta_{2}(\ell)$ in Fig. 4, and plot $\epsilon_{1,2}(\ell)$ in Fig. 5 for comparisons. One can see from Fig. 4 that, on average, and under all the same conditions (resolution, sky region, and apodization), our method helps to reduce the error of reconstruction by $2-3$ orders of magnitude at

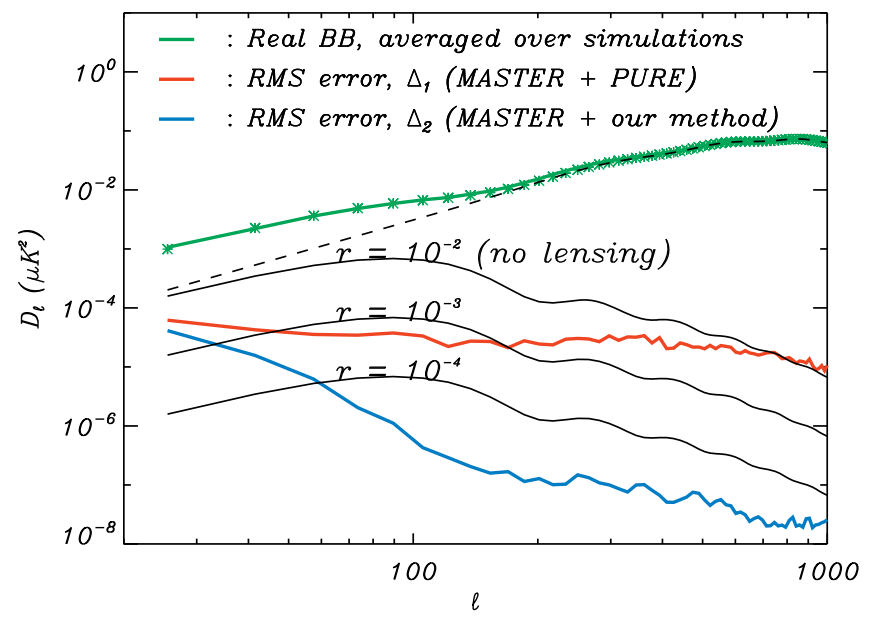

FIG. 4. Comparison of the errors of $E B$ leakage correction: $\Delta_{1}(\ell)$ for MASTER + PURE (red), and $\Delta_{2}(\ell)$ for MASTER + our method (blue); see Eqs. (1)-(2) and Sec. III B for details. Several lines are added for comparison including: the input BBspectrum of $r=0.05$ (green asterisks), the spectrum reconstructed from the real B-mode in the available region and averaged over simulations (green solid), the expected primordial $B$-mode spectra for $r=10^{-2}-10^{-4}$ (black solid), and the lensing $B$-mode spectrum (black dashed). One can see that our method helps to reduce the error of reconstruction by $2-3$ orders of magnitudes under the same conditions (resolution, simulated maps, sky region, apodization, etc.).

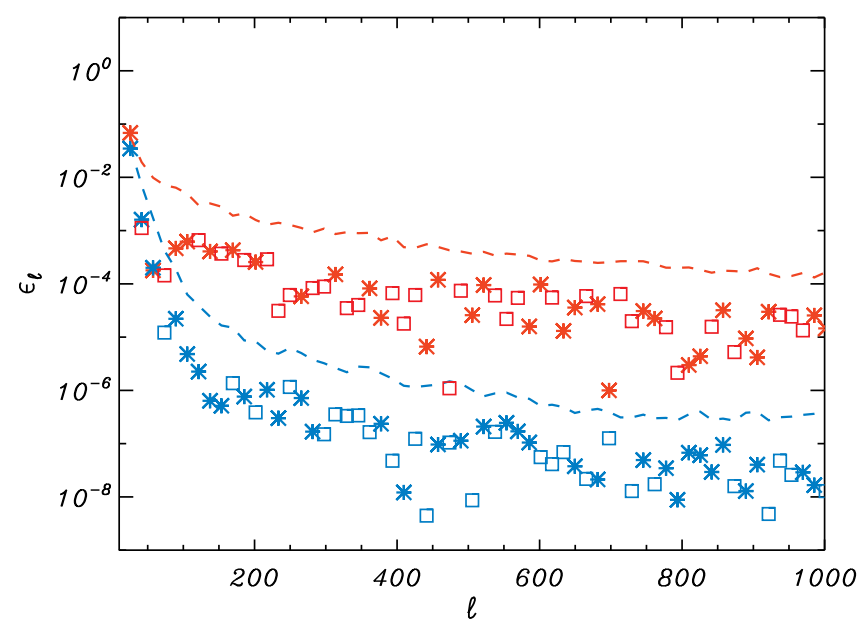

FIG. 5. Comparison of the normalized average offsets $\epsilon_{1,2}(\ell)$ [see Eq. (3)]. Red for MASTER + PURE and blue for MASTER + our method. The amplitudes of the normalized RMS are also shown in dash lines. In both cases, they are roughly 1 order of magnitude higher than the average offsets. The simulations are the same ones in Fig. 4. Note that this is a logarithmic plot, so we use asterisks and squares to mark positive negative values respectively.

higher $\ell$ with respect to the MASTER + PURE scheme. One can also see from Fig. 4 that the MASTER + PURE method gives uncertainties in the leakage correction at roughly the level of $r \approx 10^{-3}$ for the first peak and $r \approx 10^{-2}$ for higher multipoles, whereas, by an improvement of $2-3$ orders of magnitude, our method ensures that the $E B$ leakage is suppressed down to a level of $r \approx 10^{-4}-10^{-5}$ for both the first peak and higher multipoles. ${ }^{4}$ In Fig 5, the normalized average offsets $\epsilon_{1,2}(\ell)$ for MASTER + PURE and MASTER + our method are compared with each other and with the normalized rms. One can see that, in the conditions studied here, our method not only gives lower rms, but also gives lower average offsets. Meanwhile, in both cases, the average offsets are roughly 1 order of magnitude lower than the corresponding rms, so they are both statistically compatible with zero.

Note that in this section we used only $N_{\text {side }}=512$. As stated before, a higher $N_{\text {side }}$ could help to further improve the EB-leakage correction [12]. Also note that in Fig. 4, the "C1" apodization from NAMASTER with a default $10^{\circ}$ apodization length was used, without any optimization for either our method or the pure method. Although this choice is suboptimal, according to Fig. 17 of [41], optimizing the apodization of the PURE method provides improvements of less than 1 order of magnitude, whereas, in the section below, we show in Fig. 6 that optimizing the apodization of our method can provide up to 2 orders of

\footnotetext{
${ }^{4}$ We emphasize that this statement holds only for uncertainties arising from $E B$ leakage, and other issues such as sufficient foreground removal, noise, delensing, sampling uncertainties, etc., provide additional sources of error.
} 

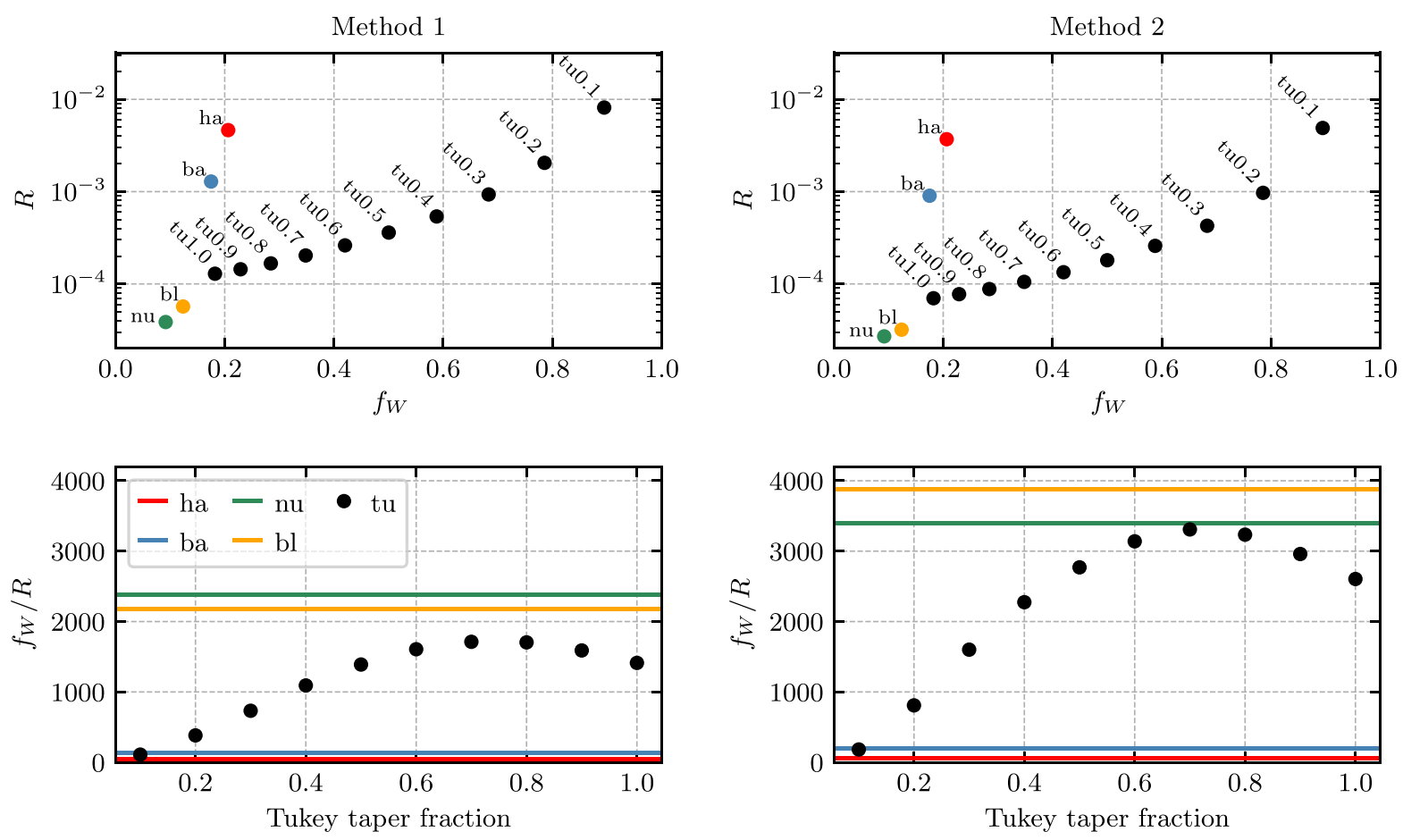

FIG. 6. Upper panels: comparison of different window functions in terms of $f_{W}$ (Eq. (4) and $R$ (Eq. (5). The labels are defined in Sec. III C. Bigger $f_{W}$ is preferred because it keeps more signal power (retaining more information), while smaller $R$ is preferred because it means lower residual error of correction; note that the recycling method gives roughly $50 \%$ lower error than method 1 . Lower panels: ratio $f_{W} / R$ as a function of the taper fraction of Tukey windows, where higher values mean better overall performance.

magnitude improvement. Therefore, after optimization is taken into account, we expect our method to be relatively even better. However, we acknowledge that in realistic applications, there will be more complicated mask shapes, and no final conclusion can be drawn about the optimal choice of apodization yet.

\section{Optimization of the posterior pixel domain apodization}

We already know from Figs. 1-3 that the residual $E B$ leakage after correction is most significant at the edge of the available sky region, and it therefore can be further suppressed by applying a posterior apodization/window function, where with posterior we mean that the apodization is applied independently of and after the pixel domain $E B$ leakage correction. Generally speaking, a more aggressive apodization gives further suppression of the residual leakage, but at the same time, the overall signal strength is reduced. In this section we test different window functions to show how to find a balance between higher signal and lower residual for the EB-leakage. We use the same mask as in the two previous subsections as well as Planck FFP9 simulations with $r=0.05$.

Given a symmetric one-dimensional window function defined on the unit interval, $w(x)$, where $0 \leq x \leq 1$, we construct its corresponding two-dimensional window function on the available region by

$$
W(n)=w\left(\frac{d(n)}{2 d_{\max }}\right),
$$

where $d(n)$ is the distance from the $n$th pixel to the edge of the mask, and $d_{\max }$ is the maximum such distance over all pixels in the available region. Such a definition ensures that the pixel domain window function is 0 at the edge and 1 at the points that are most distant to the edge. The types of $w(x)$ are chosen from the following (the abbreviations in brackets are to be used in Fig. 6):

(i) Hamming (ha) and Tukey windows with taper fractions in increments of 0.1 (tu0.1, etc.) [53]

(ii) Bartlett window (ba) [54]

(iii) Nuttall window (nu) [55]

(iv) Exact Blackman window (bl) $[53,56]$.

Note that the Tukey window is also known as the tapered cosine window; the conventional cosine window, also known as the Hann window, is recovered with a taper fraction of 1.0 ("tu1.0").

To evaluate the aggressiveness of each window function, we calculate

$$
f_{W}=\frac{1}{N} \sum_{n=1}^{N} W^{2}(n),
$$

where $N$ is the total number of pixels in the available region. $f_{W}$ is an effective measure of the sky-fraction, 
normalized such that a top-hat mask gives $f_{W}=1$. More aggressive windows remove more power, and we have $f_{W} \in[0,1]$.

Given a window/apodization function, the amplitude of the residual leakage after correction is estimated by $R$, defined as the rms of the relative error, averaged over a range of multipoles for all simulations, as follows:

$$
R=\sqrt{\frac{1}{N_{\mathrm{sim}} \cdot \Delta \ell} \cdot \sum_{\ell=\ell_{1}}^{\ell_{2}} \sum_{i=1}^{N_{\mathrm{sim}}}\left[\frac{\left(\tilde{C}_{\ell}^{B B}\right)_{i}-\left(\tilde{C}_{\ell}^{B B, c}\right)_{i}}{\left(\tilde{C}_{\ell}^{B B}\right)_{i}}\right]^{2}},
$$

where $\left(\tilde{C}_{\ell}^{B B}\right)_{i}$ and $\left(\tilde{C}_{\ell}^{B B, c}\right)_{i}$ are the pseudopower spectra of the apodized real and corrected $B$ maps for the $i$ th simulation, and $\Delta \ell=\ell_{2}-\ell_{1}+1$. The multipole range used here is $\left(\ell_{1}, \ell_{2}\right)=(60,120)$, including the recombination bump of the $B B$ spectrum.

We evaluate a set of 14 standard window functions, including ten Tukey windows with taper fractions in increments of 0.1 . For each window, we plot $R$ vs $f_{W}$, and the results for method 1 and method 2 are both shown in Fig. 6. It is seen that method 2 gives smaller residual error (lower $R$ ) than method 1 for each posterior apodization. Furthermore, we also plot the ratio $f_{W} / R$ for the different window functions. This ratio is a simple measure of the overall performance of each window. According to this, for the mask under investigation, Tukey windows with a taper fraction of around 0.7, as well as Nuttall and Blackman windows, seem to give the best EB-leakage correction in both method 1 and 2 .

\section{DISCUSSION}

In this work, we presented two methods (Secs. II A-II B) that both are capable of correcting the $E B$ leakage in the pixel domain. With emphasis on one of them, various tests showed the effectiveness of these corrections, e.g., the residual error is 2-3 orders of magnitude lower than an implementation of a conventional method (the MASTER + PURE scheme). These results are obtained using simple rectangular and circular masks, and they illustrate the potential improvements possible with this new method. The idea of pixel domain $E B$ leakage correction is based on the idea of $E B$-family decomposition previously proposed in [27,57], which herewith is proved to be an extremely useful framework for the study of polarization maps.

The advantages of a correction in pixel space are many. In Sec. III B our $E B$ leakage correction method was combined with MASTER, a pseudo- $C_{l}$ method for the reconstruction of a full-sky power spectrum. We demonstrated that the results obtained are orders of magnitude better than without explicit leakage correction. Our method provides the possibility to be combined with any pseudo- $C_{l}$ or maximum likelihood method to improve their ability for $B$-mode power spectrum reconstruction.
In addition, as shown in Sec. III C, it is possible to further reduce the error of power spectrum reconstruction by optimizing the posterior apodization applied to the corrected $B$ map; see Fig. 6 . We there explained how to use the large library of one-dimensional window functions from digital signal processing in CMB science, which provides an easy way to explore variations two dimensional window functions.

Note that although the rectangular and circular masks studied here are appropriate for characterizing the general features of the method, they are simpler than those encountered in practice. More complicated shapes will result in higher leakage and residuals. Therefore, in the future the residuals and the bias of the method should be evaluated using simulations with realistic masks particular to a certain experiment, in order to fully understand the method's performance on the application in question.

The $E B$ leakage is driven more by large scale structures than by small scale structures, since small scale structures are locally more confined and therefore do not propagate as far. Hence, a satisfactory correction of $E B$ leakage only requires the $E$-mode to be much larger than the $B$-mode at large scales, which is always true for the $\mathrm{CMB}$-also if noise is added, given that the noise is subdominant compared with the $E$-mode signal at large scales, which will be the case for upcoming CMB missions.

The methods also enable an easy treatment of noise in power spectrum reconstructions, because in the pixel domain noise and CMB simply are added linearly to make up the total signal, and our correction methods are also linear. Therefore, the $B$-mode residual $\Delta \boldsymbol{B}$ after correction is simply

$$
\Delta \boldsymbol{B}=\Delta \boldsymbol{B}_{\mathrm{CMB}}+\Delta \boldsymbol{B}_{\text {noise }} .
$$

In general, further removal of the noise in the pixel domain is impossible; however, if one assumes that the noise is Gaussian and uncorrelated with the CMB, then the two residual terms in Eq. (6) are independent, which means their cross covariance does not contribute to the overall covariance matrix. With this assumption, one can easily remove the noise contribution to the angular spectra using one of the standard methods, by using, e.g., cross spectra [58], noise spectrum models [59], null maps obtained from two half-mission maps [60], or null maps obtained from two subbands [61]. Examples of EB-leakage correction in the presence of noise can be found in Appendix E.

To our knowledge, these two methods are the first attempt to provide solutions to the $E B$ leakage in the pixel domain with negligible computational time cost. The five main obstacles in the detection of CMB $B$-modes are foreground removal, delensing, noise, systematics, and the $E B$ leakage. The present method to overcome the last also enables the more reliable investigation of $B$-mode morphology in a local sky region, opening up possibilities to have a closer look at the remaining obstacles. 


\section{ACKNOWLEDGMENTS}

We sincerely thank the anonymous referee for the helpful comments. This research has made use of data product from the Planck [62] Collaboration, the HEALPIX [63] and HEALPY packages, and the NAMASTER/PYMASTER package [51,52]. This work was partially funded by the Danish National Research Foundation (DNRF) through establishment of the Discovery Center and the Villum Fonden through the Deep Space project. Hao Liu is also supported by the National Natural Science Foundation of China (Grants No. 11653002, No. 11653003), the Strategic Priority Research Program of the CAS (Grant No. XDB23020000) and the Youth Innovation Promotion Association, CAS.

\section{APPENDIX A: TWO FORMS OF E/B DECOMPOSITION}

\section{The $\mathbf{E}$ and $B$ maps}

Here we briefly review the definition of $E(\boldsymbol{n})$ and $B(\boldsymbol{n})$ maps. The Stokes parameters $Q$ and $U$ can be decomposed into spin \pm 2 spherical harmonics $[19,21]$ as follows:

$$
Q(\boldsymbol{n}) \pm i U(\boldsymbol{n})=\sum_{l, m} a_{ \pm 2, \ell m \pm 2} Y_{\ell m}(\boldsymbol{n}),
$$

where ${ }_{ \pm 2} Y_{\ell m}(\boldsymbol{n})$ are the spin \pm 2 spherical harmonics, and the coefficients $a_{ \pm 2, \ell m}$ are given by

$$
a_{ \pm 2, \ell m}=\int(Q(\boldsymbol{n}) \pm i U(\boldsymbol{n}))_{ \pm 2} Y_{\ell m}^{*}(\boldsymbol{n}) d \boldsymbol{n} .
$$

The $E$ - and $B$-modes in harmonic space are then formed by

$$
\begin{aligned}
& a_{E, \ell m}=-\left(a_{2, \ell m}+a_{-2, \ell m}\right) / 2, \\
& a_{B, \ell m}=i\left(a_{2, \ell m}-a_{-2, \ell m}\right) / 2,
\end{aligned}
$$

and the pixel domain representations of the $E$ - and $B$-modes are

$$
\begin{aligned}
& E(\boldsymbol{n})=\sum a_{E, \ell m} Y_{\ell m}(\boldsymbol{n}), \\
& B(\boldsymbol{n})=\sum a_{B, \ell m} Y_{\ell m}(\boldsymbol{n}) .
\end{aligned}
$$

\section{The E- and B-families}

In our work, the $E$ - and $B$-families refer to those parts of the Stokes parameters, $\left(Q_{E}, U_{E}\right)$ and $\left(Q_{B}, U_{B}\right)$, that contain only $E$ - or $B$-modes respectively, and satisfy $(Q, U) \equiv\left(Q_{E}, U_{E}\right)+\left(Q_{B}, U_{B}\right)$. They are defined as follows:

\footnotetext{
${ }^{5}$ Note that in an early version of [27], there were misprints in some signs. The equations here have been corrected.
}

$$
\begin{aligned}
& \left(\begin{array}{c}
Q_{E} \\
U_{E}
\end{array}\right)(\boldsymbol{n})=\int\left(\begin{array}{cc}
G_{1} & +G_{2} \\
+G_{3} & G_{4}
\end{array}\right)\left(\boldsymbol{n}, \boldsymbol{n}^{\prime}\right)\left(\begin{array}{c}
Q \\
U
\end{array}\right)\left(\boldsymbol{n}^{\prime}\right) d \boldsymbol{n}^{\prime} \\
& \left(\begin{array}{c}
Q_{B} \\
U_{B}
\end{array}\right)(\boldsymbol{n})=\int\left(\begin{array}{cc}
G_{4} & -G_{3} \\
-G_{2} & G_{1}
\end{array}\right)\left(\boldsymbol{n}, \boldsymbol{n}^{\prime}\right)\left(\begin{array}{c}
Q \\
U
\end{array}\right)\left(\boldsymbol{n}^{\prime}\right) d \boldsymbol{n}^{\prime},
\end{aligned}
$$

where the $G_{1-4}$ functions are defined as

$$
\begin{aligned}
& G_{1}\left(\boldsymbol{n}, \boldsymbol{n}^{\prime}\right)=\sum_{l, m} F_{+, \ell m}(\boldsymbol{n}) F_{+, \ell m}^{*}\left(\boldsymbol{n}^{\prime}\right), \\
& G_{2}\left(\boldsymbol{n}, \boldsymbol{n}^{\prime}\right)=\sum_{l, m} F_{+, \ell m}(\boldsymbol{n}) F_{-, \ell m}^{*}\left(\boldsymbol{n}^{\prime}\right), \\
& G_{3}\left(\boldsymbol{n}, \boldsymbol{n}^{\prime}\right)=\sum_{l, m} F_{-, \ell m}(\boldsymbol{n}) F_{+, \ell m}^{*}\left(\boldsymbol{n}^{\prime}\right), \\
& G_{4}\left(\boldsymbol{n}, \boldsymbol{n}^{\prime}\right)=\sum_{l, m} F_{-, \ell m}(\boldsymbol{n}) F_{-, \ell m}^{*}\left(\boldsymbol{n}^{\prime}\right),
\end{aligned}
$$

and the $F_{+,-}$functions are defined in terms of the spin-2 spherical harmonics as

$$
\begin{aligned}
& F_{+, \ell m}(\boldsymbol{n})=-\frac{1}{2}\left[{ }_{2} Y_{\ell m}(\boldsymbol{n})+{ }_{-2} Y_{\ell m}(\boldsymbol{n})\right], \\
& F_{-, \ell m}(\boldsymbol{n})=-\frac{1}{2 i}\left[{ }_{2} Y_{\ell m}(\boldsymbol{n})-{ }_{-2} Y_{\ell m}(\boldsymbol{n})\right] .
\end{aligned}
$$

Note that $G_{i}$ are real and $G_{2}=G_{3}$.

The $E$ - and $B$-families can also be conveniently calculated by setting $a_{l m}^{B}$ or $a_{l m}^{E}$ to zero, and running a standard inverse transform using HEALPIX. For more details of these two families, see [27,57], also discussed in [64].

\section{APPENDIX B: DETAILED INTRODUCTION OF METHODS 1 AND 2}

\section{Method 1: Diffusive inpainting}

In [22], it was shown that the ambiguous mode $\psi$ which represents the mixing between the localized $E$ and $B$ estimators satisfies the spherical bi-Laplacian equation,

$$
\nabla^{2}\left(\nabla^{2}+2\right) \psi=0
$$

subject to homogeneous Neumann and Dirichlet boundary conditions at the edge of the known region. Assuming that the power in the $E$-mode dominates the power in the $B$-mode, the purified $B$-mode, calculated by removing the ambiguous mode $\psi$ from the corrupted $B$-mode, is a good approximation to the true $B$-mode.

We here simplify the approach by replacing the biLaplacian equation by the Laplacian equation and neglecting the Neumann boundary conditions. ${ }^{6}$ In this case, a simple numerical solution is the relaxation method. It is

\footnotetext{
${ }^{6}$ The solutions of the simplified Laplacian problem retain the basic large-scale structure of the bi-Laplacian solutions; however, small-scale structures can be neglected.
} 
important to note that it is possible to work also with standard full-sky definitions of the $E$ - and $B$-mode, which is more convenient.

It was shown by [28] that the $E B$ leakage is most significant at the edge of the mask, which naturally provides a reliable boundary condition. Therefore, it is tempting to solve the $E B$ leakage by relaxation methods using a boundary constraint. This is implemented by diffusive inpainting $[25,26]$, in which sky pixels ${ }^{7}$ are iteratively replaced by the average of their neighbors, except for the pixels on the boundary. This results in a zero Laplacian solution that is subject to the given boundary condition.

Therefore, method 1 runs as introduced in Sec. II A.

\section{Method 2: Recycling the E-mode}

While the method introduced above provides a means to roughly remove smoothly distributed leakage, we here suggest a second method which also accounts for smaller scale structure well inside the unmasked region. Since in this method, the corrupted component will be reused for correction, it will be referred to as the "recycling method."

Before describing the method we introduce our notation. As discussed in [27,57] and briefly reviewed in Appendix A 2, the polarized sky signal can be decomposed into the $E$ - and $B$-families as

$$
(Q, U)=\left(Q_{E}, U_{E}\right)+\left(Q_{B}, U_{B}\right),
$$

where $\left(Q_{E}, U_{E}\right)$ stems only from the $E$-mode, and $\left(Q_{B}, U_{B}\right)$ only from the $B$-mode. This decomposition forms the basis of the recycling method.

Consider a sky map, divided into two regions as shown by Fig. 7. Its polarization signal can be decomposed according to Eq. (B2). Conventionally the polarization vector is introduced as $\boldsymbol{P}=(Q, U)$, such that $\left(Q_{E}, U_{E}\right)$ and $\left(Q_{B}, U_{B}\right)$ can be denoted as $\boldsymbol{P}_{E}$ and $\boldsymbol{P}_{B}$. In the following we wish to describe $\boldsymbol{P}_{E}$ and $\boldsymbol{P}_{B}$ in region 1 and 2 separately, which suggests the obvious notation $\boldsymbol{P}_{E_{1,2}}$ and $\boldsymbol{P}_{B_{1,2}}$. However, for visual simplicity we shorten the notation as follows:

$$
\begin{array}{ll}
\boldsymbol{E}_{1} \equiv \boldsymbol{P}_{E_{1}} \equiv\left(Q_{E}, U_{E}\right)_{1} ; & \boldsymbol{B}_{1} \equiv \boldsymbol{P}_{B_{1}} \equiv\left(Q_{B}, U_{B}\right)_{1} ; \\
\boldsymbol{E}_{2} \equiv \boldsymbol{P}_{E_{2}} \equiv\left(Q_{E}, U_{E}\right)_{2} ; & \boldsymbol{B}_{2} \equiv \boldsymbol{P}_{B_{2}} \equiv\left(Q_{B}, U_{B}\right)_{2} .
\end{array}
$$

Each Stokes component of $\boldsymbol{E}_{i}$ and $\boldsymbol{B}_{i}$ should be regarded as a full-sky map whose values at pixels outside region $i$ are zero. Therefore, the sum $\boldsymbol{E}_{1}+\boldsymbol{B}_{1}+\boldsymbol{E}_{2}+\boldsymbol{B}_{2}$ again forms the input polarized sky maps $\boldsymbol{P}$. Both $\boldsymbol{E}$ and $\boldsymbol{B}$ arise from integrating $\boldsymbol{P}$ over the full sky. In this context, each of the

\footnotetext{
${ }^{7}$ For this we have used the HEALPIX package (http://healpix .sourceforge.net) and therefore adopted their pixelization scheme. However, the method is not tailored to function with that pixelization only.
}

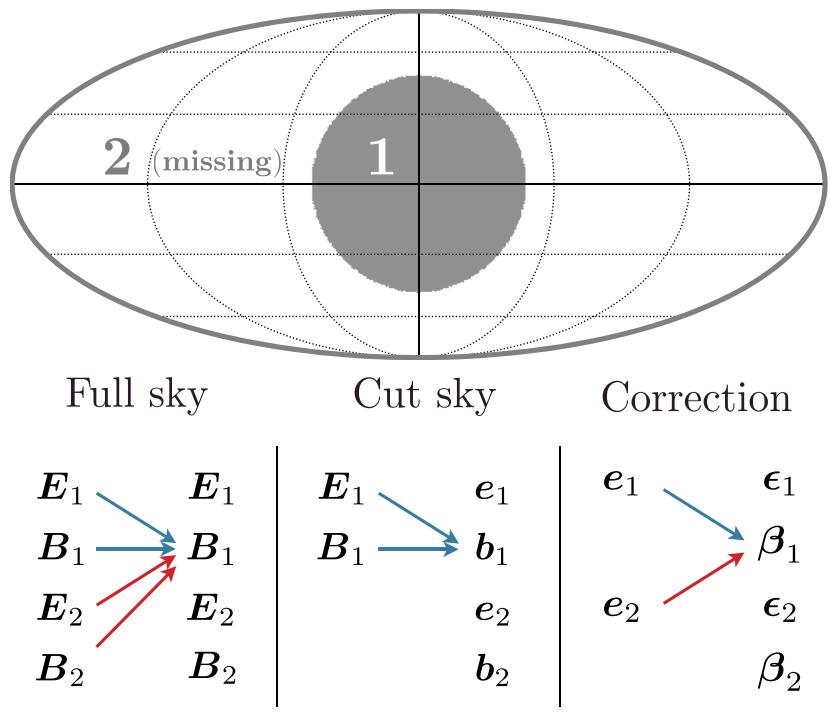

FIG. 7. Illustration of recycling method, see Sec. II B for explanations. Arrows denote contributions from one quantity to another, a notation adopted for the equations in the text.

quantities in the sum, while describing only part of the sky, can be thought of as receiving contributions from all, $\boldsymbol{E}_{1}$, $\boldsymbol{B}_{1}, \boldsymbol{E}_{2}$, and $\boldsymbol{B}_{2}$. The lower-left panel in Fig. 7 illustrates this process, and in line with this sketch, we introduce a symbolic notation in which the contributing terms are denoted with arrows. We write the corresponding equation describing the contributions to quantity $\boldsymbol{X}$ as

$\boldsymbol{X} \equiv\left(\boldsymbol{E}_{1} \rightarrow \boldsymbol{X}\right)+\left(\boldsymbol{B}_{1} \rightarrow \boldsymbol{X}\right)+\left(\boldsymbol{E}_{2} \rightarrow \boldsymbol{X}\right)+\left(\boldsymbol{B}_{2} \rightarrow \boldsymbol{X}\right)$,

where $\boldsymbol{X}$ can stand for either of $\boldsymbol{E}_{1}, \boldsymbol{B}_{1}, \boldsymbol{E}_{2}$ or $\boldsymbol{B}_{2}$, and in the following we will refer to a bracketed term as a contributor. Note that since in practice, region 2 will be the missing part of the sky (due to either a mask or incomplete observation of the sky), $\boldsymbol{E}_{2}$ and $\boldsymbol{B}_{2}$ will only be used in the following discussion, but not in any of the computations presented later. In this notation we are able to define a set of rules (Appendix C) providing detailed relations between contributors. In this framework we describe the $E B$ leakage, and we study relations among the contributors to arrive at a solution for its correction.

If only region 1 is available, the contributions of $\boldsymbol{E}_{2}$ and $\boldsymbol{B}_{2}$ obviously disappear, as indicated in the lower middle panel of Fig. 7. Consequently, in Eq. (B4) the third and fourth terms disappear, and Eq. (B4) reduces to

$$
\boldsymbol{x} \equiv\left(\boldsymbol{E}_{1} \rightarrow \boldsymbol{x}\right)+\left(\boldsymbol{B}_{1} \rightarrow \boldsymbol{x}\right)
$$

where $\boldsymbol{x}$ can be either of $\boldsymbol{e}_{1}, \boldsymbol{b}_{1}, \boldsymbol{e}_{2}$ or $\boldsymbol{b}_{2}$. Quantities arising from these incomplete sums are denoted by lower-case latin letters and are what we previously referred to as corrupted. 
Obviously, these quantities are generally different from the corresponding real quantities $\boldsymbol{X}$.

Focusing on the corrupted component $\boldsymbol{b}_{1}$, the two contributors that form it are $\left(\boldsymbol{E}_{1} \rightarrow \boldsymbol{b}_{1}\right)$ and $\left(\boldsymbol{B}_{1} \rightarrow \boldsymbol{b}_{1}\right)$, as marked by the blue arrows in the lower, middle panel of Fig. 7. These two contributors have distinct meanings: $\left(\boldsymbol{B}_{1} \rightarrow \boldsymbol{b}_{1}\right)$ contains the $B$-to- $B$ deformation, which can be corrected in the angular power spectrum, e.g., by the MASTER method [32]; the contributor $\left(\boldsymbol{E}_{1} \rightarrow \boldsymbol{b}_{1}\right)$ is the $E B$ leakage. It is this term which we attempt to correct for in this work. However, since $\boldsymbol{E}_{1}$ is unknown in the case of partial sky coverage, the true leakage term $\left(\boldsymbol{E}_{1} \rightarrow \boldsymbol{b}_{1}\right)$ is generally not available. Nevertheless, with some approximations, we shall show how to remove this leakage in the pixel domain to a highly sufficient degree.

We hereto restrict ourselves to the $E$-family output of the cut-sky case, $\boldsymbol{e}_{1}$ and $\boldsymbol{e}_{2}$, which together form a full-sky map of the $E$-family. We decompose this map again in terms of $E$ - and $B$-families, as shown in the lower-right panel of Fig. 7. However, this will not produce any $B$-family output (except for numerical and pixelization errors), which in terms of the contributors is written as

$$
\begin{gathered}
\boldsymbol{\beta}_{1}=\left(\boldsymbol{e}_{1} \rightarrow \boldsymbol{\beta}_{1}\right)+\left(\boldsymbol{e}_{2} \rightarrow \boldsymbol{\beta}_{1}\right)=0 ; \\
-\left(\boldsymbol{e}_{1} \rightarrow \boldsymbol{\beta}_{1}\right)=\left(\boldsymbol{e}_{2} \rightarrow \boldsymbol{\beta}_{1}\right) \neq 0 .
\end{gathered}
$$

Here and in the figure, lower-case greek letters denote the $E$ - and $B$-families from the corrupted maps $\boldsymbol{e}_{1}+\boldsymbol{e}_{2}$.

In the CMB, $E$-modes clearly dominate over $B$-modes. The observation ${ }^{8}$ that then $\boldsymbol{E}_{1} \approx \boldsymbol{e}_{1}$ enables us to reason that the morphology of the $E B$ leakage term $\left(\boldsymbol{E}_{1} \rightarrow \boldsymbol{b}_{1}\right)$ is well approximated by the contributor $\left(\boldsymbol{e}_{1} \rightarrow \boldsymbol{\beta}_{1}\right)$. In fact, we expect an approximate proportionality between the two contributors (see Appendix D) such that the $E$-to- $B$ leakage can be corrected in the pixel domain by linearly removing the contributor $\left(\boldsymbol{e}_{1} \rightarrow \boldsymbol{\beta}_{1}\right)$ from $\boldsymbol{b}_{1}$. In short, we recycle a product of one corrupted component, $\boldsymbol{e}_{1}$, for the correction of another, $\boldsymbol{b}_{1}$.

Therefore, method 2 runs as introduced in Sec. II B.

\section{APPENDIX C: THE SYMBOLIC SYSTEM BEHIND THE RECYCLING METHOD}

The description of the recycling method presented in Appendix B 2 is based on a purely symbolic representation. In this formulation, we here provide a complete set of relations among the quantities. For convenience and consistency, we continue to use the notation from Eq. (B3).

Firstly we have the total conservation rules, which were given in Eq. (B4) and rewritten below,

\footnotetext{
${ }^{8}$ For the two cases discussed in Sec. II C we find correlation coefficients of 0.99 and 0.97 , respectively.
}

$\boldsymbol{X} \equiv\left(\boldsymbol{E}_{1} \rightarrow \boldsymbol{X}\right)+\left(\boldsymbol{B}_{1} \rightarrow \boldsymbol{X}\right)+\left(\boldsymbol{E}_{2} \rightarrow \boldsymbol{X}\right)+\left(\boldsymbol{B}_{2} \rightarrow \boldsymbol{X}\right)$,

where $\boldsymbol{X}$ is one of $\boldsymbol{E}_{1}, \boldsymbol{B}_{1}, \boldsymbol{E}_{2}$ or $\boldsymbol{B}_{2}$.

As presented in Eq. (B6), the contributors presented in Sec. II B satisfy the orthogonality rules, which can be written as

$$
\left(\boldsymbol{X}_{i} \rightarrow \boldsymbol{Y}_{i}\right)+\left(\boldsymbol{X}_{j} \rightarrow \boldsymbol{Y}_{i}\right)=0,
$$

where $\boldsymbol{X}$ and $\boldsymbol{Y}$ are either $\boldsymbol{E}$ or $\boldsymbol{B}$, but not the same; and $i, j$ are either 1 or 2 but not the same. For convenience, we extend Eq. (C2) as follows:

$$
\begin{aligned}
& \left(\boldsymbol{E}_{1} \rightarrow \boldsymbol{B}_{1}\right)+\left(\boldsymbol{E}_{2} \rightarrow \boldsymbol{B}_{1}\right)=0, \\
& \left(\boldsymbol{E}_{1} \rightarrow \boldsymbol{B}_{2}\right)+\left(\boldsymbol{E}_{2} \rightarrow \boldsymbol{B}_{2}\right)=0, \\
& \left(\boldsymbol{B}_{1} \rightarrow \boldsymbol{E}_{1}\right)+\left(\boldsymbol{B}_{2} \rightarrow \boldsymbol{E}_{1}\right)=0, \\
& \left(\boldsymbol{B}_{1} \rightarrow \boldsymbol{E}_{2}\right)+\left(\boldsymbol{B}_{2} \rightarrow \boldsymbol{E}_{2}\right)=0 .
\end{aligned}
$$

These rules follow from the orthogonality between the $E$ - and $B$-families.

Combining the total conservation rules with the orthogonality rules, one gets the inner conservation rules as follows:

$$
\left(\boldsymbol{X}_{i} \rightarrow \boldsymbol{X}_{i}\right)+\left(\boldsymbol{X}_{j} \rightarrow \boldsymbol{X}_{i}\right)=\boldsymbol{X}_{i},
$$

where $\boldsymbol{X}$ is either $\boldsymbol{E}$ or $\boldsymbol{B}$, and $i, j$ are either 1 or 2 but not the same. Again we extend this equation for convenience as

$$
\begin{aligned}
& \left(\boldsymbol{E}_{1} \rightarrow \boldsymbol{E}_{1}\right)+\left(\boldsymbol{E}_{2} \rightarrow \boldsymbol{E}_{1}\right)=\boldsymbol{E}_{1}, \\
& \left(\boldsymbol{E}_{1} \rightarrow \boldsymbol{E}_{2}\right)+\left(\boldsymbol{E}_{2} \rightarrow \boldsymbol{E}_{2}\right)=\boldsymbol{E}_{2}, \\
& \left(\boldsymbol{B}_{1} \rightarrow \boldsymbol{B}_{1}\right)+\left(\boldsymbol{B}_{2} \rightarrow \boldsymbol{B}_{1}\right)=\boldsymbol{B}_{1}, \\
& \left(\boldsymbol{B}_{1} \rightarrow \boldsymbol{B}_{2}\right)+\left(\boldsymbol{B}_{2} \rightarrow \boldsymbol{B}_{2}\right)=\boldsymbol{B}_{2} .
\end{aligned}
$$

When one connects the full-sky quantities $(\boldsymbol{E}$ or $\boldsymbol{B})$ with cut-sky quantities ( $\boldsymbol{e}$ or $\boldsymbol{b}$ ), one has the completeness rules as follows:

$$
\begin{aligned}
& \boldsymbol{E}_{1}+\boldsymbol{B}_{1}=\boldsymbol{e}_{1}+\boldsymbol{b}_{1}, \\
& \boldsymbol{E}_{2}+\boldsymbol{B}_{2}=\boldsymbol{e}_{2}+\boldsymbol{b}_{2},
\end{aligned}
$$

which follow from the completeness of the spin-2 spherical harmonics. In particular, when region 2 is unavailable, we have

$$
\boldsymbol{E}_{2}+\boldsymbol{B}_{2}=\boldsymbol{e}_{2}+\boldsymbol{b}_{2}=0 .
$$

All $E$-to- $E, B$-to- $B, E$-to- $B$, and $B$-to- $E$ leakages can be formally described by, and are also subject to, the symbolic system represented by Eqs. (C1)-(C7). Note that the symbolic system in this section does not contain any 
approximation. The approximation needed for the recycling method is contained in Eq. (D7) in Appendix D.

Alternatively, all rules presented here can also be expressed in terms of the equations in Appendix A 2. With the definitions,

$G_{E} \equiv\left(\begin{array}{cc}G_{1} & +G_{2} \\ +G_{3} & G_{4}\end{array}\right) ; \quad G_{B} \equiv\left(\begin{array}{cc}G_{4} & -G_{3} \\ -G_{2} & G_{1}\end{array}\right)$,

we can express the contributors as, e.g.,

$$
\begin{gathered}
\boldsymbol{X}=\int G_{\boldsymbol{X}}\left(\boldsymbol{n}, \boldsymbol{n}^{\prime}\right) \boldsymbol{P}\left(\boldsymbol{n}^{\prime}\right) d \boldsymbol{n}^{\prime} \\
\boldsymbol{x}=\int_{1} G_{\boldsymbol{X}}\left(\boldsymbol{n}, \boldsymbol{n}^{\prime}\right) \boldsymbol{P}\left(\boldsymbol{n}^{\prime}\right) d \boldsymbol{n}^{\prime} \\
\left(\boldsymbol{X}_{i} \rightarrow \boldsymbol{Y}_{j}\right)=\left[\int_{i} G_{\boldsymbol{Y}}\left(\boldsymbol{n}, \boldsymbol{n}^{\prime}\right) \boldsymbol{X}\left(\boldsymbol{n}^{\prime}\right) d \boldsymbol{n}^{\prime}\right]_{j}
\end{gathered}
$$

where $\int_{i}$ denotes the integration over region $i$ only, and $[\ldots]_{j}$ denotes the restriction of the evaluated quantity in region $j$.

\section{APPENDIX D: ABOUT THE LINEAR FITTING FOR RECYCLING METHOD}

In the recycling method, we use linear fitting to determine the factor that connects the template $\left(\boldsymbol{e}_{1} \rightarrow \boldsymbol{\beta}_{1}\right)$ to the real leakage $\left(\boldsymbol{E}_{1} \rightarrow \boldsymbol{b}_{1}\right)$. Here we provide more details on why this can be done by linear fitting. have

For a cut sky map, only region 1 is available; thus we

$$
\boldsymbol{E}_{1}+\boldsymbol{B}_{1}=\boldsymbol{e}_{1}+\boldsymbol{b}_{1}
$$

Assuming there is no initial $B$-mode (like the assumption in Sec. III A), or $B \ll E$ and $B$ therefore can be neglected, then we have

$$
\boldsymbol{E}_{1}=\boldsymbol{e}_{1}+\boldsymbol{b}_{1} .
$$

Since now there is only one input component $\boldsymbol{E}_{1}$, we automatically get

$$
\begin{aligned}
& \boldsymbol{e}_{1}=\left(\boldsymbol{E}_{1} \rightarrow \boldsymbol{e}_{1}\right), \\
& \boldsymbol{b}_{1}=\left(\boldsymbol{E}_{1} \rightarrow \boldsymbol{b}_{1}\right) .
\end{aligned}
$$

Since there is no signal in region 2, we also have [cf. Eq. (C7)]

$$
\boldsymbol{e}_{2}+\boldsymbol{b}_{2}=0
$$

however, note that at the same time we have $\boldsymbol{e}_{2} \neq 0$ and $\boldsymbol{b}_{2} \neq 0$.
Equations (D4)-(D5) tell us that

$$
\begin{aligned}
\boldsymbol{b}_{1} & \equiv\left(\boldsymbol{e}_{1} \rightarrow \boldsymbol{b}_{1}\right)+\left(\boldsymbol{b}_{1} \rightarrow \boldsymbol{b}_{1}\right)+\left(\boldsymbol{e}_{2} \rightarrow \boldsymbol{b}_{1}\right)+\left(\boldsymbol{b}_{2} \rightarrow \boldsymbol{b}_{1}\right) \\
& =\left(\boldsymbol{e}_{1} \rightarrow \boldsymbol{b}_{1}\right)+\left(\boldsymbol{b}_{1} \rightarrow \boldsymbol{b}_{1}\right)=\left(\boldsymbol{E}_{1} \rightarrow \boldsymbol{b}_{1}\right) .
\end{aligned}
$$

Since the contributor $\left(\boldsymbol{b}_{1} \rightarrow \boldsymbol{b}_{1}\right)$ represents the $B$-to- $B$ leakage, we can expect it to have similar morphology to the input $B$-mode even with a mask. Thus we have

$$
\left(b_{1} \rightarrow b_{1}\right) \underset{\sim}{\propto} b_{1},
$$

where $\propto$ stands for approximate proportionality. Thus according to Eq. (D6), as long as the amplitudes of $\boldsymbol{b}_{1}$ and $\left(\boldsymbol{b}_{1} \rightarrow \boldsymbol{b}_{1}\right)$ are not close to each other (which is observed), we find

$$
\left(\boldsymbol{e}_{1} \rightarrow \boldsymbol{b}_{1}\right) \underset{\sim}{\propto}\left(\boldsymbol{E}_{1} \rightarrow \boldsymbol{b}_{1}\right) .
$$

Finally, two contributors are always equal when the input components, destination regions and output types are all equal; thus we have

$$
\left(\boldsymbol{e}_{1} \rightarrow \boldsymbol{\beta}_{1}\right)=\left(\boldsymbol{e}_{1} \rightarrow \boldsymbol{b}_{1}\right) \underset{\sim}{\propto}\left(\boldsymbol{E}_{1} \rightarrow \boldsymbol{b}_{1}\right) .
$$

Since $\left(\boldsymbol{e}_{1} \rightarrow \boldsymbol{\beta}_{1}\right)$ is our template, and $\left(\boldsymbol{E}_{1} \rightarrow \boldsymbol{b}_{1}\right)$ is the real $E B$ leakage, Eq. (D9) says that one can use linear fitting to connect the template to the real leakage in the recycling method.

It is also easy to explain why we choose $\left(\boldsymbol{e}_{1} \rightarrow \boldsymbol{\beta}_{1}\right)$ as the template, but not $\left(\boldsymbol{b}_{1} \rightarrow \boldsymbol{\beta}_{1}\right)$ : when the input $B$-mode is not zero, $\left(\boldsymbol{b}_{1} \rightarrow \boldsymbol{\beta}_{1}\right)$ will contain the real input $B$-mode, but $\left(\boldsymbol{e}_{1} \rightarrow \boldsymbol{\beta}_{1}\right)$ will not.

\section{APPENDIX E: THE EB-LEAKAGE WITH NOISE}

Here we reproduce Figs. 4-5 by adding white noise with 1 and $10 \mu \mathrm{K} \cdot \operatorname{arcmin}$ amplitudes to each simulation, respectively. We run the test in a completely blind way, so each program, either MASTER + PURE or MASTER+ our method, is unaware of the presence of noise. Thus the resulting angular power spectrum will automatically include the noise contribution and become higher than the primordial one. It is important to notice that, according to Eqs. (1)-(2), in a blind test, the error of reconstruction will automatically use the noisy map as reference; i.e., the noise itself is regarded as a natural part of the input map, and will not be removed here. The advantage of this approach is that it helps us to focus on the EB-leakage itself.

The results of the tests with noise are presented in Fig. 8, where one can see that the green line becomes higher with higher noise, but the error of EB-leakage correction for both methods is still reasonably small, so they both give fairly stable EB-leakage correction with/without noise. We also notice that, when noise increases, the error of EBleakage correction increases more slowly for our method than for MASTER + PURE. 

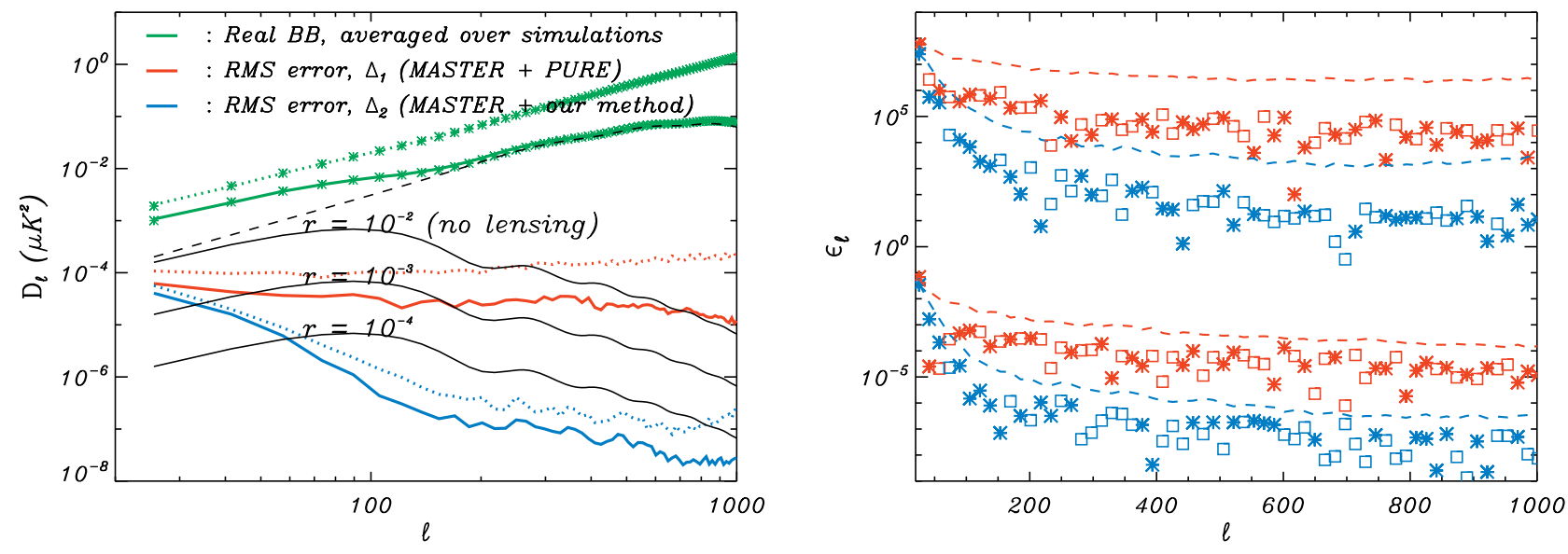

FIG. 8. Left: Same as Fig. 4, only that white noise with 1 and $10 \mu \mathrm{K} \cdot \operatorname{arcmin}$ amplitudes (solid/dotted) is added to each simulation respectively. Note that the test is run in a blind way, so noise is regarded as natural part of the input maps. Right: The corresponding $\epsilon_{1,2}(\ell)$, similar to Fig. 5. The $10 \mu \mathrm{K} \cdot \operatorname{arcmin}$ noise results are up-shifted to make the lines visible.

[1] P. A. R. Ade, N. Aghanim, M. I. R. Alves, C. ArmitageCaplan, M. Arnaud, M. Ashdown, F. Atrio-Barandela, J. Aumont, H. Aussel et al. (Planck Collaboration), Astron. Astrophys. 571, A1 (2014).

[2] R. Adam, P. A. R. Ade, N. Aghanim, Y. Akrami, M. I. R. Alves, F. Arguieso, M. Arnaud, F. Arroja, M. Ashdown et al. (Planck Collaboration), Astron. Astrophys. 594, A1 (2016).

[3] Y. Akrami, F. Arroja, M. Ashdown, J. Aumont, C. Baccigalupi, M. Ballardini, A. J. Banday, R. B. Barreiro, N. Bartolo et al. (Planck Collaboration), arXiv:1807.06205.

[4] M. Hazumi, J. Borrill, Y. Chinone, M. A. Dobbs, H. Fuke, A. Ghribi, M. Hasegawa, K. Hattori, M. Hattori, W. L. Holzapfel et al., in SPIE Astronomical Telescopes + Instrumentation, 2012, Amsterdam, Netherlands (2012), Vol. 8442 of Proc. SPIE, p. 844219.

[5] K. N. Abazajian, P. Adshead, Z. Ahmed, S. W. Allen, D. Alonso, K. S. Arnold, C. Baccigalupi, J. G. Bartlett, N. Battaglia, B. A. Benson et al., arXiv:1610.02743.

[6] B. Keating, S. Moyerman, D. Boettger, J. Edwards, G. Fuller, F. Matsuda, N. Miller, H. Paar, G. Rebeiz, I. Schanning et al., arXiv:1110.2101.

[7] J. A. Rubiño-Martín, R. Rebolo, M. Aguiar, R. GénovaSantos, F. Gómez-Reñasco, J. M. Herreros, R. J. Hoyland, C. López-Caraballo, A. E. Pelaez Santos, V. S. de la Rosa et al., Proc. SPIE Int. Soc. Opt. Eng. 8444, 84442Y (2012).

[8] H. Li, S.-Y. Li, Y. Liu, Y.-P. Li, Y. Cai, M. Li, G.-B. Zhao, C.-Z. Liu, Z.-W. Li, H. Xu et al., Natl. Sci. Rev. 6, 145 (2019).

[9] P. A. R. Ade, N. Aghanim, Z. Ahmed, R. W. Aikin, K. D. Alexander, M. Arnaud, J. Aumont, C. Baccigalupi et al. (BICEP2/Keck and Planck Collaborations), Phys. Rev. Lett. 114, 101301 (2015).
[10] N. Aghanim, Y. Akrami, M. Ashdown, J. Aumont, C. Baccigalupi, M. Ballardini, A. J. Banday, R. B. Barreiro, and N. Bartolo (Planck Collaboration), arXiv:1807.06209.

[11] A. Lewis, A. Challinor, and N. Turok, Phys. Rev. D 65, 023505 (2001).

[12] E. F. Bunn, M. Zaldarriaga, M. Tegmark, and A. de OliveiraCosta, Phys. Rev. D 67, 023501 (2003).

[13] A. Lewis, Phys. Rev. D 68, 083509 (2003).

[14] E. F. Bunn, New Astron. Rev. 47, 987 (2003).

[15] M. Zaldarriaga, arXiv:astro-ph/0305272.

[16] W. Zhao and D. Baskaran, Phys. Rev. D 82, 023001 (2010).

[17] K. M. Smith, Phys. Rev. D 74, 083002 (2006).

[18] K. M. Smith and M. Zaldarriaga, Phys. Rev. D 76, 043001 (2007).

[19] M. Zaldarriaga and U.c. v. Seljak, Phys. Rev. D 55, 1830 (1997).

[20] M. Kamionkowski, A. Kosowsky, and A. Stebbins, Phys. Rev. D 55, 7368 (1997).

[21] M. Zaldarriaga, Astrophys. J. 503, 1 (1998).

[22] E. F. Bunn, Phys. Rev. D 83, 083003 (2011).

[23] M. Bucher and T. Louis, Mon. Not. R. Astron. Soc. 424, 1694 (2012).

[24] E. F. Bunn and B. Wandelt, Phys. Rev. D 96, 043523 (2017).

[25] P. A. R. Ade, N. Aghanim, C. Armitage-Caplan, M. Arnaud, M. Ashdown, F. Atrio-Barandela, J. Aumont, C. Baccigalupi, A. J. Banday et al. (Planck Collaboration), Astron. Astrophys. 571, A24 (2014).

[26] P. A. R. Ade, N. Aghanim, M. Arnaud, F. Arroja, M. Ashdown, J. Aumont, C. Baccigalupi, M. Ballardini, A. J. Banday et al. (Planck Collaboration), Astron. Astrophys. 594, A17 (2016).

[27] H. Liu, J. Creswell, and P. Naselsky, J. Cosmol. Astropart. Phys. 05 (2018) 059. 
[28] J. Kim and P. Naselsky, Astron. Astrophys. 519, A104 (2010).

[29] H. Liu, J. Creswell, and K. Dachlythra, J. Cosmol. Astropart. Phys. 19 (2019) 046.

[30] M. Tegmark, Phys. Rev. D 55, 5895 (1997).

[31] M. Tegmark and A. de Oliveira-Costa, Phys. Rev. D 64, 063001 (2001).

[32] E. Hivon, K. M. Górski, C. B. Netterfield, B. P. Crill, S. Prunet, and F. Hansen, Astrophys. J. 567, 2 (2002).

[33] G. Efstathiou, Mon. Not. R. Astron. Soc. 348, 885 (2004).

[34] J. Jewell, S. Levin, and C. H. Anderson, Astrophys. J. 609, 1 (2004).

[35] B. D. Wandelt, D. L. Larson, and A. Lakshminarayanan, Phys. Rev. D 70, 083511 (2004).

[36] H. K. Eriksen, I. J. O’Dwyer, J. B. Jewell, B. D. Wandelt, D. L. Larson, K. M. Górski, S. Levin, A. J. Banday, and P. B. Lilje, Astrophys. J. Suppl. 155, 227 (2004).

[37] G. Polenta, D. Marinucci, A. Balbi, P. de Bernardis, E. Hivon, S. Masi, P. Natoli, and N. Vittorio, J. Cosmol. Astropart. Phys. 11 (2005) 001.

[38] R. Saha, P. Jain, and T. Souradeep, Astrophys. J. Lett. 645, L89 (2006).

[39] P. Abrial, Y. Moudden, J.-L. Starck, J. Fadili, J. Delabrouille, and M. K. Nguyen, Stat. Methodol. 5, 289 (2008).

[40] K. T. Inoue, P. Cabella, and E. Komatsu, Phys. Rev. D 77, 123539 (2008).

[41] J. Grain, M. Tristram, and R. Stompor, Phys. Rev. D 79, 123515 (2009).

[42] A. Gruppuso, A. de Rosa, P. Cabella, F. Paci, F. Finelli, P. Natoli, G. de Gasperis, and N. Mandolesi, Mon. Not. R. Astron. Soc. 400, 463 (2009).

[43] J. B. Jewell, H. K. Eriksen, B. D. Wandelt, I. J. O'Dwyer, G. Huey, and K. M. Górski, Astrophys. J. 697, 258 (2009).

[44] A. Zacchei, D. Maino, C. Baccigalupi, M. Bersanelli, A. Bonaldi, L. Bonavera, C. Burigana, R. C. Butler, F. Cuttaia, G. de Zotti et al., Astron. Astrophys. 536, A5 (2011).

[45] J. Kim, P. Naselsky, and N. Mandolesi, Astrophys. J. Lett. 750, L9 (2012).

[46] J.-L. Starck, M. J. Fadili, and A. Rassat, Astron. Astrophys. 550, A15 (2013).
[47] D. Molinari, A. Gruppuso, G. Polenta, C. Burigana, A. De Rosa, P. Natoli, F. Finelli, and F. Paci, Mon. Not. R. Astron. Soc. 440, 957 (2014).

[48] P. A. R. Ade, N. Aghanim, M. Arnaud, M. Ashdown, J. Aumont, C. Baccigalupi, A. J. Banday, R. B. Barreiro, J. G. Bartlett et al. (Planck Collaboration), Astron. Astrophys. 594, A12 (2016).

[49] The GreenPol website at UCSB, https://www.deepspace .ucsb.edu/projects/greenpol.

[50] The GreenPol website at NBI, https://deep-space.nbi.ku.dk/.

[51] D. Alonso, J. Sanchez, and A. Slosar (LSST Dark Energy Science), Mon. Not. R. Astron. Soc. 484, 4127 (2019).

[52] D. Alonso, J. Sanchez, and A. Slosar, Namaster: A unified pseudo-cl framework, https://github.com/LSSTDESC/ NaMaster.

[53] F. J. Harris, Proc. IEEE 66, 51 (1978).

[54] J. K. Gautam, A. Kumar, and R. Saxena, IEEE Trans. Signal Process. 44, 2098 (1996).

[55] A. Nuttall, IEEE Trans. Acoust., Speech, Signal Process. 29, 84 (1981).

[56] R. B. Blackman and J. W. Tukey, Bell Syst. Tech. J. 37, 185 (1958).

[57] H. Liu, Astron. Astrophys. 617, A90 (2018).

[58] G. Hinshaw, D. N. Spergel, L. Verde, R. S. Hill, S. S. Meyer, C. Barnes, C. L. Bennett, M. Halpern, N. Jarosik, A. Kogut et al., Astrophys. J. Suppl. 148, 135 (2003).

[59] P. Ade et al. (Planck Collaboration), Astron. Astrophys. 571, A15 (2014).

[60] N. Aghanim, M. Arnaud, M. Ashdown, J. Aumont, C. Baccigalupi, A. J. Banday, R. B. Barreiro, J. G. Bartlett, N. Bartolo et al. (Planck Collaboration), Astron. Astrophys. 594, A11 (2016).

[61] G. Hinshaw, C. Barnes, C. L. Bennett, M. R. Greason, M. Halpern, R. S. Hill, N. Jarosik, A. Kogut, M. Limon, S. S. Meyer et al., Astrophys. J. Suppl. 148, 63 (2003).

[62] The Planck data release, (2018), https://www.cosmos.esa .int/web/planck/pla.

[63] K. M. Górski, E. Hivon, A. J. Banday, B. D. Wandelt, F. K. Hansen, M. Reinecke, and M. Bartelmann, Astrophys. J. 622, 759 (2005).

[64] A. Rotti and K. Huffenberger, J. Cosmol. Astropart. Phys. 01 (2019) 045. 